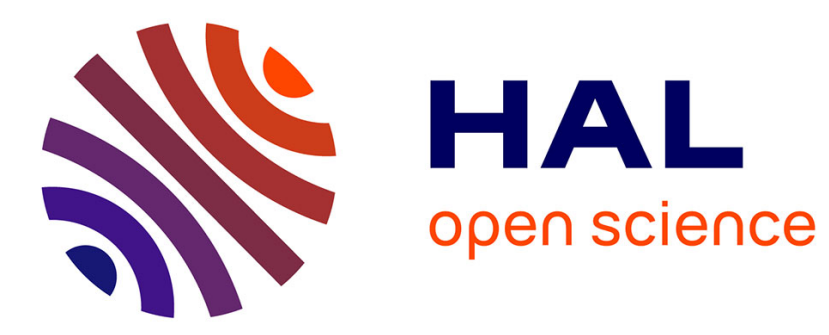

\title{
Local Electric Field Variation in the Field Emission Process
}

\author{
A. Esperidião, N. de Oliveira, C. de Castilho
}

\section{To cite this version:}

A. Esperidião, N. de Oliveira, C. de Castilho. Local Electric Field Variation in the Field Emission Process. Journal de Physique IV Proceedings, 1996, 06 (C5), pp.C5-59-C5-64. 10.1051/jp4:1996509 . jpa-00254388

\section{HAL Id: jpa-00254388 https://hal.science/jpa-00254388}

Submitted on 1 Jan 1996

HAL is a multi-disciplinary open access archive for the deposit and dissemination of scientific research documents, whether they are published or not. The documents may come from teaching and research institutions in France or abroad, or from public or private research centers.
L'archive ouverte pluridisciplinaire HAL, est destinée au dépôt et à la diffusion de documents scientifiques de niveau recherche, publiés ou non, émanant des établissements d'enseignement et de recherche français ou étrangers, des laboratoires publics ou privés. 


\title{
Local Electric Field Variation in the Field Emission Process
}

\author{
A.S.C. Esperidião, N.B. de Oliveira and C.M.C. de Castilho \\ Instituto de Física, Universidade Federal da Bahia, Campus Universitário da Federação, \\ 40210-340 Salvador, Bahia, Brazil
}

\begin{abstract}
Tunnelling probabilities in field emission processes are usually calculated assuming flat emitting surfaces. This corresponds to consider the electric field as a constant along the potential barrier. Previous work have shown the importance of taking into account image effects but neglecting local field variations. Even when the local field has been taken into account, a proper three dimensional calculation of the transmission coefficient was not performed. We discuss previous calculation models, their limitations and conclusions, comparing them with our model results. We have determined the transmission coefficient using a 3-D numerical integration process. Considering a completely planar surface and another one with an hemispherical protrusion superimposed on it, we could compare the relative influence of either the local field variation along the potential barrier and image effects. These results lead to the conclusion that corrections due to the local field variation are of a magnitude that deserves to be considered, together as those resulting from the image potential.
\end{abstract}

\section{INTRODUCTION}

Electron tunnelling probabilities for cold emission from metals has been theoretically treated by several authors since the first description of the phenomena in $1897 / 1 \%$. In the seminal paper by Fowler and Nordheim $/ 2 /$ "the first clear picture of the mechanism" $/ 3 /$ was presented. On that paper, the solution of Schrödinger's equation was performed together with the consideration of the Fermi-Dirac statistics. Calculations of ref. 2 are one-dimensional with the potential barrier being just the one resulting from the superposition of a constant potential, equal to the work function inside the metal, and the linear form of the potential for a constant external field. A refinement of the first approach was done by Nordheim $/ 4 /$ for inclusion of image effects, with corections to this later presented by Burguess et al. $15 /$. In both cases a completelly flat geometry is assumed for the metal surface,so that the electric field intensity is constant along the potential barrier. The local enhancement of the electric field, resulting from surface irregularities was taken into account by Bono and Good $/ 6 /$, with particular application to the resolving power of the field ion microscope.

The aim of this work is to discuss the relative influence of image effects and of the local enhancement of the electric field in tunnelling probabilities for electrons emitted from metal tips. For this we have used a numerical approach were the field variation along the barrier for the tunnelling electron is taken in account. 


\section{MODELS FOR THE POTENTIAL ENERGY AND WKB CALCULATIONS}

We have considered a few models for the electric potential energy. The simplest one is for a flat metal surface, e.g. which coincides with the XOY plane, and an external constant field, F. Inside the metal the potential energy is the work function, $\phi$, so that, for a tunnelling electron with the Fermi energy, we can write:

$$
\begin{aligned}
& U=-\phi, \text { for } z<0 \\
& U=-e F z, \text { for } z>0
\end{aligned}
$$

where $\mathrm{e}$ is the elementary (positive) charge.

The consideration above is strictly one dimensional. An improvement to this would to consider the possibility of tunnelling along direction $\theta$ (see figure 1) and perform an integration in $\theta$ from 0 to $\pi / 2$ what corresponds to a reduction in the effective field along the barrier. This leads to eqs. 1 becomming

$$
\begin{aligned}
& U=-\phi, \text { for } z<0 \\
& U=-e F r \cos \theta, \text { for } z>0
\end{aligned}
$$

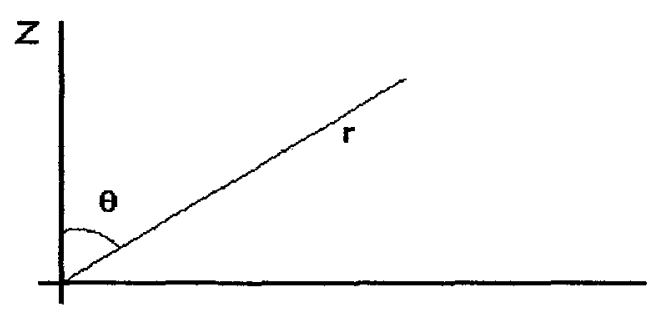

Figure 1 - Direction $\theta$ with respect to normal of the sample's surface.

If we include, into the previous model, the effect of the electron image charge we have:

$$
\begin{aligned}
& \mathrm{U}=-\phi, \text { for } \mathrm{z}<0 \\
& \mathrm{U}=-\mathrm{e} \mathrm{Fr} \cos \theta-\left(1 / 4 \pi \varepsilon_{\mathrm{o}}\right) \mathrm{e}^{2}[1-\exp (-\lambda \mathrm{r} \cos \theta)] /(4 \mathrm{r} \cos \theta), \\
& \text { for } \mathrm{z}>0
\end{aligned}
$$

In eq. 3.b the factor $[1-\exp (-\lambda r \cos \theta)]$ is an usual solution $/ 7 /$ to avoid the divergence at points very close to the surface. The requirement of continuity in the potential energy at the metal surface gives:

$$
\lambda=\left(4 \pi \varepsilon_{\mathrm{o}} / \mathrm{e}^{2}\right) 4 \phi
$$

In order to model a local field variation we have considered a planar surface with an hemispherical protrusion superimposed on it $/ 6,8 /$. The energy resulting from the superposition of the plane plus hemispherical protrusion is then:

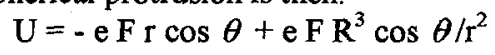

The simultaneous consideration of the hemispherical protrusion and image effects leads to the adoption of three image charges in order to assure a constant potential at the sample's surface. Figure 2 shows the position of the image charges which, for the region where $z>0$, corresponds to a potential energy given by:

$$
\mathrm{U}=-\mathrm{e} F \mathrm{r} \cos \theta+\mathrm{e} F \mathrm{R}^{3} \cos \theta / \mathrm{r}^{2}+\mathrm{U}_{\mathrm{i}, 1}+\mathrm{U}_{\mathrm{i}, 2}+\mathrm{U}_{\mathrm{i}, 3}
$$

where the terms $U_{i, j}$ for $j=1,2$ and 3 correspond, respectively, to the contributions of the image charges 1 , 2 and 3 , as shown in fig. 2 .

$$
\begin{aligned}
& \mathrm{U}_{\mathrm{i}, 1}=\left(1 / 4 \pi \varepsilon_{\mathrm{o}}\right) \mathrm{e}^{2}[1-\exp (-\lambda \mathrm{r} \cos \theta)] /(4 \mathrm{r} \cos \theta) \\
& \mathrm{U}_{\mathrm{i}, 2}=\left(1 / 4 \pi \varepsilon_{0}\right) \mathrm{e}^{2} \mathrm{R} /\left[2\left(\mathrm{r}^{2}-\mathrm{R}^{2}\right)\right](1-\exp [-\lambda(\mathrm{r}-\mathrm{R})] \\
& \mathrm{U}_{\mathrm{i}, 3}=\left(1 / 4 \pi \varepsilon_{\mathrm{o}}\right) \mathrm{e}^{2} \mathrm{R} /\left\{2\left[\mathrm{r}^{4}+\mathrm{R}^{4}+2 \mathrm{R}^{2} \mathrm{r}^{2}\left(2 \cos ^{2} \theta-1\right)\right]^{1 / 2}\right\}
\end{aligned}
$$

The analytic determination of the transmission coefficient, which results from the solution of Schrödinger's equation, depends on the adopted approach for solving this equation and on the necessary 


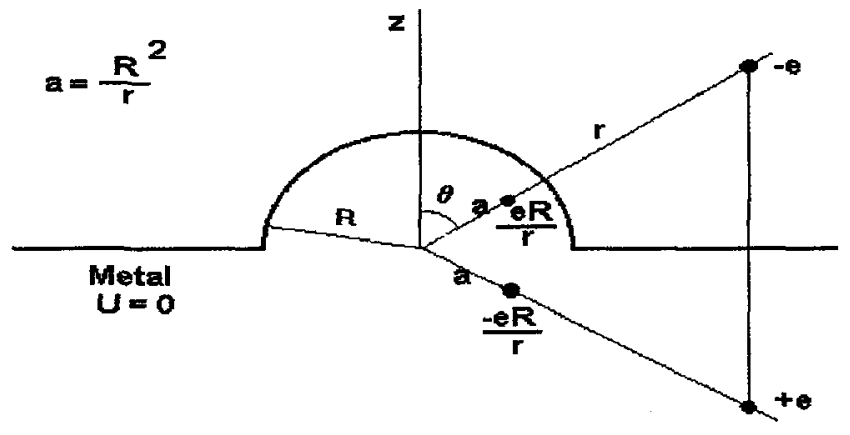

Figure 2 - Image charges for the model of a planar surface plus a superimposed hemisphere.

approximations resulting from the particular form of the potential energy. We have considered the models above mentioned for the electron tunnelling barrier, within the limits of the WKB approximation /9/. Thus, the electrons' wave functions in the metal are expressed by plane waves approaching the metal-vacuum interface normally with an energy equal to the Fermi energy. Even in cases where the interface was not considered as planar, this normal incidence was locally taken into account, the effect of curvature just affecting the transmission coefficient through the field direction along the potential barrier. The WKB expression for the transmission coefficient in one dimension is given by:

$$
\rho=\mathrm{A}^{2} \exp \left\{-2 \int_{z_{0}}^{z_{1}}\left[\left(2 \mathrm{~m} / \hbar^{2}\right)^{1 / 2}(\mathrm{U}-\mathrm{E})^{1 / 2} \mathrm{dz}\right]\right\}
$$

where $\mathrm{A}^{2}$ is related to the rate of tunnelling atempts and $\mathrm{z}_{0}$ and $\mathrm{z}_{1}$ are the classical turning points of the potential barrier (U - E ). A three dimensional version of the WKB approach / 10 / is obtained by summing one-dimensional transmission rates from infinitely small solid angles, which leads to:

$$
\rho=\mathrm{A}^{2} \int_{\theta=0}^{\pi / 2} \int_{\phi=0}^{2 \pi} \exp \left\{-2 \int_{z_{0}}^{z_{1}}\left[\left(2 \mathrm{~m} / \hbar^{2}\right)^{1 / 2}(\mathrm{U}-\mathrm{E})^{1 / 2} \mathrm{dz}\right] \sin \theta \mathrm{d} \theta \mathrm{d} \phi\right\}
$$

The difficulty in having an analytical solution for eqs. 7 or 8 results from the form of the potential energy $U$, implying then on the adoption of a numerical solution. We have numerically solved eq. 8 for the models of potential energy cited above where, in case of the models corresponding to eqs. 5 and 6 , the field was taken as variable along the potential barrier. Figure 3 presents results for the transmission coefficient using models of eqs. $2,3,5$ and 6 . It is possible to observe that corrections resulting from the inclusion of the local field enhancement are almost of the same importance as those resulting from image effects. Traditional numerical calculations for the transmission coefficient do not include variation of the field along the potential barrier. The importance of doing so can be inferred from table I where we express the transmission coefficient as a function of $F_{0}$ (which corresponds to the field close to the plane and far away from the protrusion, when it is the case). We can see then that neglection of the local enhancement of field leads to a too low value for the transmission coeffiecient. On the other hand if we take, along the potential barrier, the same field at the very apex of a half sphere protrusion we obtain a value extremelly high. Even consideration of an average value (between $3 F_{0}$ and $F_{0}$ ) is not adequate if we compare with the "real value" of the field for case of an hemisphere superimposed on a plane surface.

Table I - Transmission coefficient for different values of the field $F_{0}$. Column 2 corresponds to a variable field along the potential barrier for an hemisphere of radius equal to $1,5 \mathrm{~A}$, superimposed on a planar surface.

\begin{tabular}{|c|c|c|c|c|}
\hline$F_{0}(\mathrm{~V} / \mathrm{A})$ & Constant $\mathrm{F}=\mathrm{F}_{0}$ & Plane + Half Sphere & Constant $\mathrm{F}=2 \mathrm{~F}_{0}$ & Constant $\mathrm{F}=3 \mathrm{~F}_{0}$ \\
\hline 0.4 & $0.28 \times 10^{-7}$ & $0.52 \times 10^{-6}$ & $0.18 \times 10^{-3}$ & $0.38 \times 10^{-2}$ \\
\hline 0.8 & $0.18 \times 10^{-3}$ & $0.24 \times 10^{-2}$ & $0.18 \times 10^{-1}$ & $0.94 \times 10^{-1}$ \\
\hline 1.2 & $0.38 \times 10^{-2}$ & $0.38 \times 10^{-1}$ & $0.94 \times 10^{-1}$ & $0.30 \times 10^{0}$ \\
\hline
\end{tabular}




\section{IS THE WKB A REASONABLE APPROACH?}

The previous calculation shows how important is to consider the local enhancement of the electric field (besides image charge effects) when we try to model the potential barrier for field emission. However, at least one question remains: till what extent is the WKB approach adequate for tunnelling processes where the field is of such intensity as the ones present in the field emission and field ionisation phenomena? This question has its importance stressed since its is known that WKB solutions are inaccurate when the potential changes abruptly $/ 11 /$. Then, there is some space to explore alternative routes in determining, respectivelly, ionisation rate constants and transmission coefficients. A possible approach (in one dimension) is the use of the so called multi-step method /12,13/ where a potential barrier is approximated by a sucession of rectangular barriers ("slices"). The transmission coefficient is then "calculated by connecting momentum eigenfunctions" /12/. Considering the model barrier as a sucession of "slices" in form of rectangular barriers of small width, the wave function in the jth "slice" can be written in the form:

$$
\psi_{j}(x)=A_{j} \exp \left(i k_{j} x\right)+B_{j} \exp \left(-i k_{j} x\right)
$$

where the coefficients are related to the "incident" wave function by

$$
\left(\begin{array}{c}
A_{j} \\
B_{j}
\end{array}\right)=\prod_{l=j-1}^{0} M_{l}\left(\begin{array}{l}
A_{0} \\
B_{0}
\end{array}\right)
$$

and

$$
\mathrm{k}_{\mathrm{j}}=\left[2 \mathrm{~m}\left(\mathrm{E}-\mathrm{U}_{\mathrm{j}}\right)\right]^{1 / 2} / \hbar
$$

Setting $A_{0}=1$ and $B_{N+1}=0$ in eq. 10 for $j=N+1$ it is possible then to obtain the transmission coefficient which is given by

$$
\mathrm{D}(\mathrm{E})=\left[\mathrm{k}_{\mathrm{N}+1} / \mathrm{k}_{0}\right]\left|A_{N+1}\right|^{2}
$$

In order to compare WKB and multi-step calculations we have used, as an example where it is possible an analytical exact solution, a barrier with the form of $C /\left[\cosh ^{2}(x / A)\right] / 14 /$. Figure 4 shows the form of the barrier for different values of $A$ and table II shows the calculated results using WKB, the exact solution /14/ and the multi-step ones. It is easily seen that results from WKB calculations become poorer when the barrier becomes steeper. In Figure 5 we present one-dimensional calculations of the transmission coefficient for electrons tunnelling from a completely flat metal surface under the effect of an external electric field. Results are obtained using the traditional calculation of Fowler and Nordheim $/ 2 /$, the multistep the ones from a simple WKB determination.

Tabel II - Transmission coefficient for a potential barrier in form of $\mathrm{C} /\left(\cosh (\mathrm{x} / \mathrm{A})^{2}\right.$ calculated by different methods.

\begin{tabular}{|c|c|c|c|}
\hline Width (A) & Multi-step & Exact Solution & WKB \\
\hline 02 & $0.133 \times 10^{-1}$ & $0.133 \times 10^{-1}$ & $0.985 \times 10^{-2}$ \\
\hline 20 & $0.885 \times 10^{-20}$ & $0.886 \times 10^{-20}$ & $0.856 \times 10^{-20}$ \\
\hline
\end{tabular}

\section{CONCLUSIONS}

We have performed numerical WKB calculations for an electron tunnelling from a metal sample under an external electric field for different models of the potential energy. These results show that, together the inclusion of image charge effects, it is important to take into account the variation of the electric field along the potential barrier. Comparison between numerical WKB calculations, another numerical method (multi-step) and the exact solution for a particular kind of potential barrier shows the inconvenience of using WKB for steep barriers. Another evidence of this is the discrepancy in the results of one-dimensional models for tunnelling electrons from a metal planar surface, as we compare F-N calculations, multi-step and WKB determination of the transmission coefficient. Hence, a possible improvement is a threedimensional extension of the multi-step method which is under way. 


\section{ACKNOWLEGMENT}

We are indebted to Dr. R. Forbes for valuable suggestions and for raising the atention for this problem to one of us (CMCdeC). This work has the financial support of UFBA and CNPq (Brazil).

\section{REFERENCES:}

1. Wood, R. W., Phys. Rev. 5, 1, 1897.

2. Fowler, R. H. and Nordheim, L., Proc. Roy. Soc. Lond., Ser. A 119, 173, 1928.

3. Good, R. H. and Müller, E. W., Encyclopedia of Physics, vol. 21 (Springer, Berlin, 1956).

4. Nordheim, L., Proc. Roy. Soc. Lond., Ser. A 121, 626, 1928.

5. Burguess, R. E, Kroemer, H. and Houston, J. M., Phys. Rev. 90, 515, 1953.

6. Bono, S. and Good Jr, R. H., Surf. Sci. 134, 272 (1983).

7. Jennings, P. J., Jones, R. O. and Weinert, M., Phys. Rev. B 37, 6113, 1988.

8. de Castilho, C. M. C. and Kingham, D. R., Surf. Sci. 173, 75, 1986.

9. Bohm, D., Quantum Theory (Prentice Hall, New York, 1951).

10. Haydock, R. and Kingham, D. R., Surf. Sci. 103, 239, 1981.

11. Messiah, A., Quantum Mechanics (North-Holland, Amsterdam, 1961).

12. Ando, Y. and Itoh, T., J. Appl. Phys. 61, 1497, 1987.

13. Renan, R., Pereira, J. M., Ribeiro, J., Freire, V. N. and Farias, G. A., Brazilian Journal of Physics, 24, 192, 1994.

14. Landau, L. D. and Lifshitz, E. M., Quantum Mechanics (Pergamon Press, Oxford, 1977). 


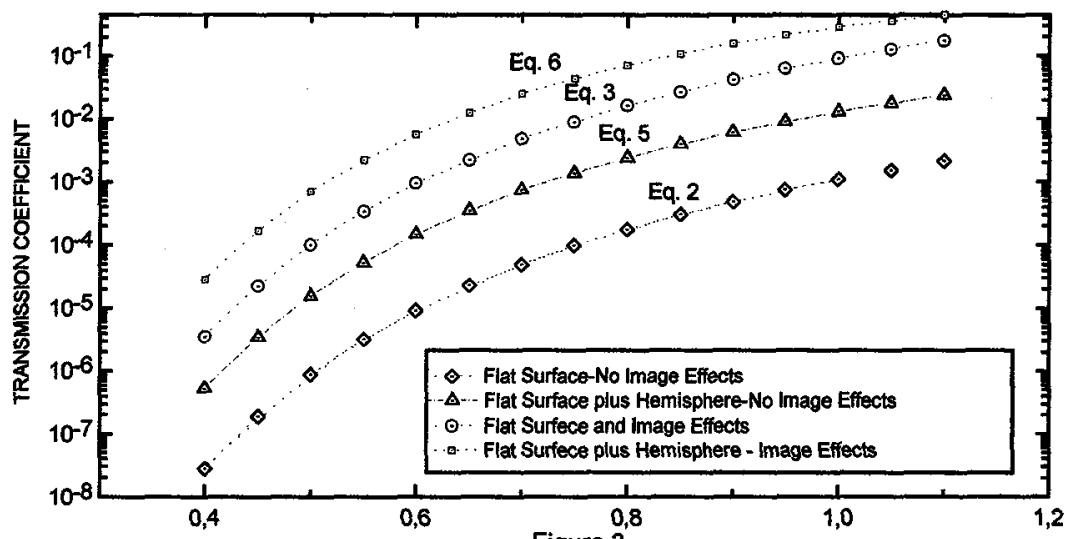

Figure 3

FIELD (VIA)

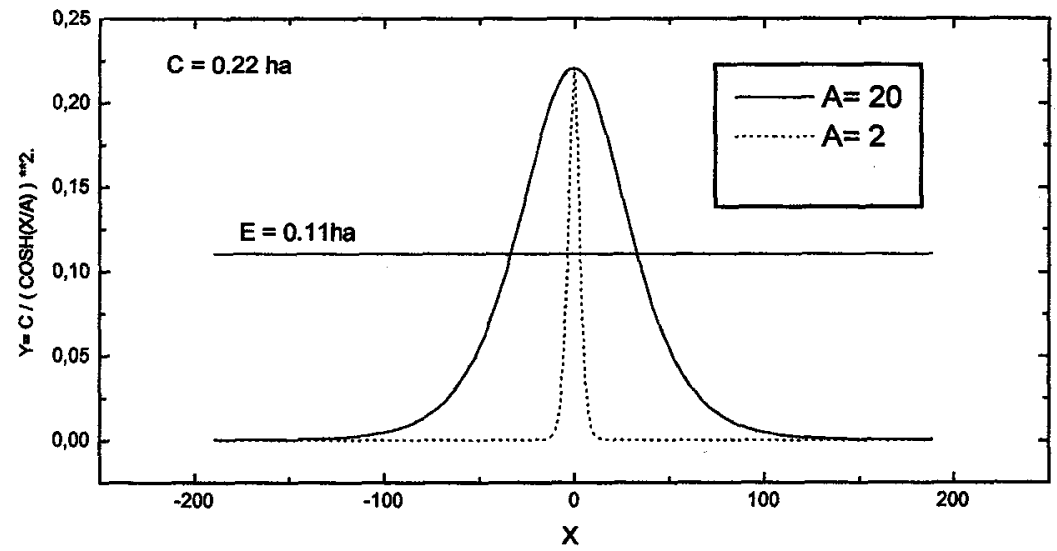

Fig. 4 - Model potential barrier corresponding to results of table II

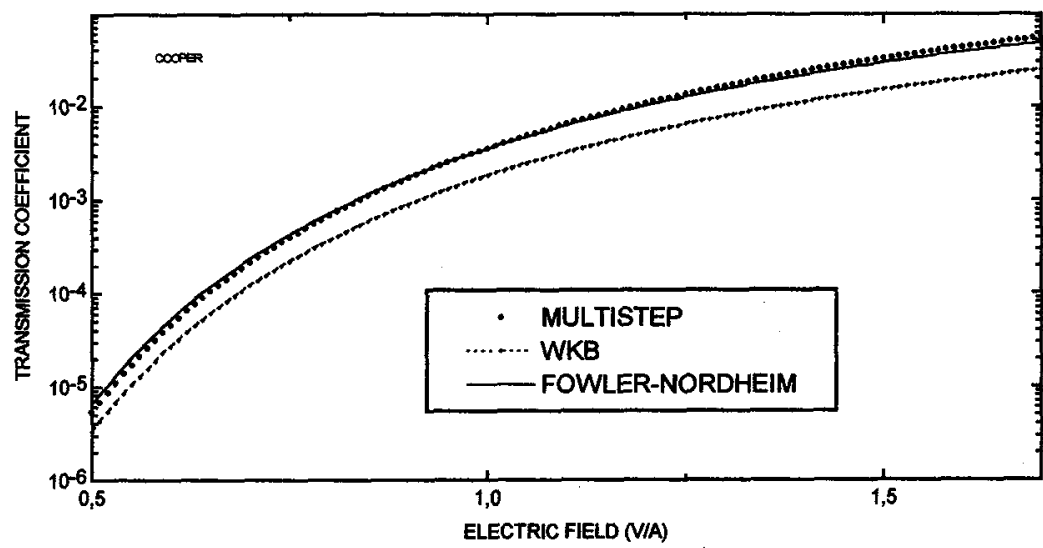

Fig. 5 - Comparison between different methods for calculating the transmission coefficient 